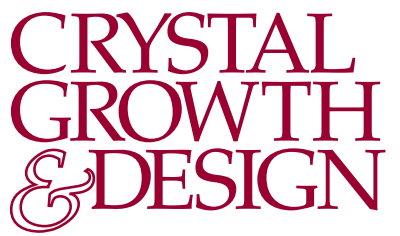

\title{
New Multicomponent Forms of the Antiretroviral Nevirapine with Improved Dissolution Performance
}

Published as part of a Crystal Growth and Design virtual special issue Remembering the Contributions and Life of Prof. Joel Bernstein

Rogeria N. Costa, ${ }^{*}{ }^{\dagger}$ Ana L. Reviglio, ${ }^{\S, \bullet}$ Sana Siedler, ${ }^{\ddagger}$ Simone G. Cardoso, ${ }^{\ddagger}$ Yamila G. Linck, ${ }^{\S, \bullet}$ Gustavo A. Monti, ${ }^{\S}$ Alexandre M. G. Carvalho, ${ }^{\perp, \# \odot ~ J a c k s o n ~ A . ~ L . ~ C . ~ R e s e n d e, " ~}$ Marcelo H. C. Chaves, ${ }^{\nabla}$ Helvécio V. A. Rocha, ${ }^{\nabla}$ Duane Choquesillo-Lazarte, ${ }^{\circledR}$ Lourdes Infantes, and Silvia L. Cuffini* ${ }^{\dagger}$

${ }^{\dagger}$ Instituto de Ciência e Tecnologia (ICT), Universidade Federal de São Paulo (UNIFESP), 12231-280 São José dos Campos, Brazil

${ }^{\S}$ FAMAF-Universidad Nacional de Córdoba, 5016, Córdoba, Argentina

- IFEG-CONICET, 5016, Córdoba, Argentina

‡Centro de Ciências da Saúde (CCS), Universidade Federal de Santa Catarina (UFSC), 88040-900 Florianópolis, Brazil

${ }^{\perp}$ Laboratório Nacional de Luz Síncrotron (LNLS), Centro Nacional de Pesquisa em Energia e Materiais (CNPEM), 13083-100

Campinas, Brazil

"Universidade Federal de Mato Grosso (UFMT), 78600-000 Barra do Garças, Brazil

${ }^{\nabla}$ Laboratório de Micro e Nanotecnologia (LMN), Farmanguinhos, Fundação Oswaldo Cruz (FIOCRUZ), 21040-361 Rio de

Janeiro, Brazil

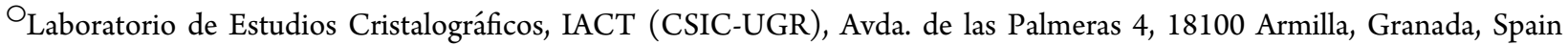

- Instituto de Química Física Rocasolano (IQFR), Consejo Superior de Investigaciones Científicas (CSIC), 28006 Madrid, Spain

Supporting Information

ABSTRACT: In the pharmaceutical area, some drugs exhibit physicochemical properties that adversely affect the formulation processes for bioavailability and effectiveness. Nevirapine (NVP) is an antiretroviral drug that presents low aqueous solubility, which directly impacts its bioavailability. Among all possible modifications, multicomponent crystals, such as cocrystals and eutectic compositions, have been successfully used to improve the solubility of drugs. In this work, the propensity of the formation of multicomponent systems of NVP with seven possible coformers were predicted and tested: salicylic acid (SA), 3-hydroxybenzoic acid (3HBZC), 4hydroxybenzoic acid (4HBZC), saccharin (SAC), theophyl-

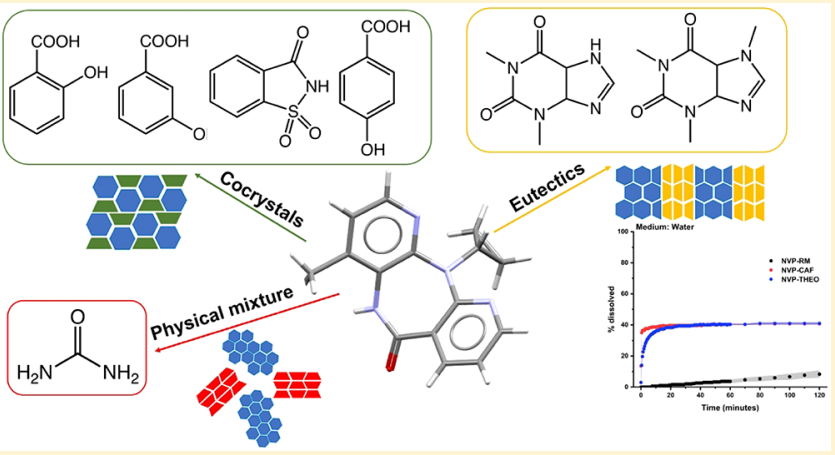
line (THEO), caffeine (CAF), and urea (URE). Results indicate that NVP-SA, NVP-SAC, NVP-3HBZC, and NVP-4HBZC are cocrystals, whereas NVP-THEO and NVP-CAF are eutectic materials, and NVP-URE is a solid physical mixture. A temperature-dependent disorder behavior was identified for NVP-SA cocrystal. Dissolution studies for the eutectic materials are reported, evidencing that these materials exhibit a significant increase in NVP dissolution kinetics.

\section{INTRODUCTION}

Nevirapine (NVP) (11-cyclopropyl-5,11-dihydro-4-methyl-6Hdipyrido[3,2-b:2', $\left.3^{\prime}-\mathrm{e}\right][1,4]$ diazepin-6-one $)^{1,2}$ is an antiretroviral drug used for the treatment of AIDS/HIV-1 infection. According to the Biopharmaceutics Classification System (BCS), NVP is classified as a Class II drug; i.e., it presents low water solubility and high permeability in the gastrointestinal tract. $^{3}$ The low water solubility is a challenge during the formulation of the drug, directly affecting its bioavailability.
High doses are often necessary to guarantee its effectiveness, increasing possible adverse side effects.

Crystallization methods can be used to obtain different crystal forms of NVP in order to improve its dissolution and, consequently, its bioavailability. Cocrystals and eutectics are

Received: August 24, 2019

Revised: December 2, 2019

Published: December 9, 2019 


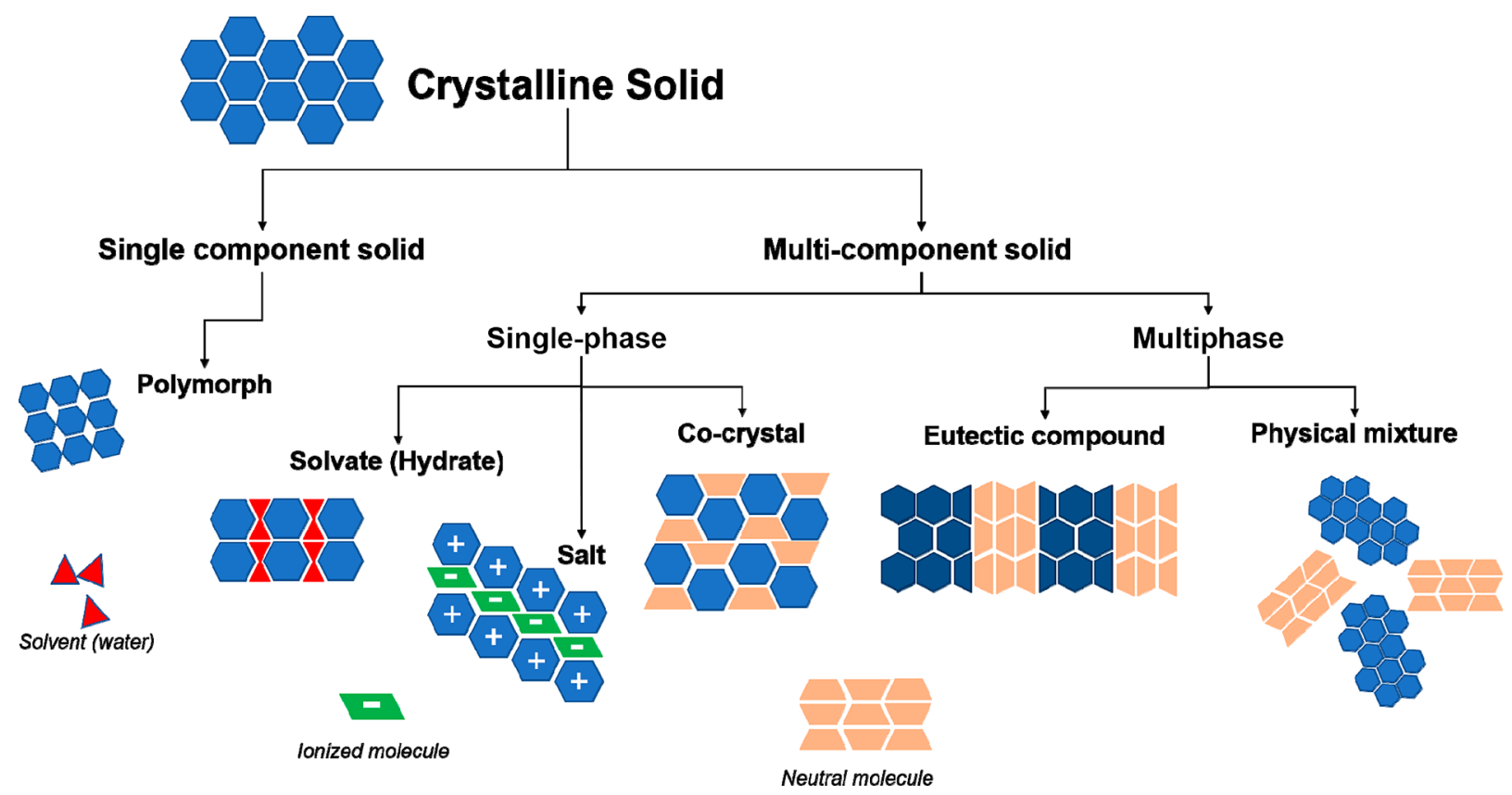

Figure 1. Representation of the structural organization in multicomponent solid forms of an API.

Scheme 1. Bidimensional Representation of the Seven Coformers Used in the Cocrystal Screening

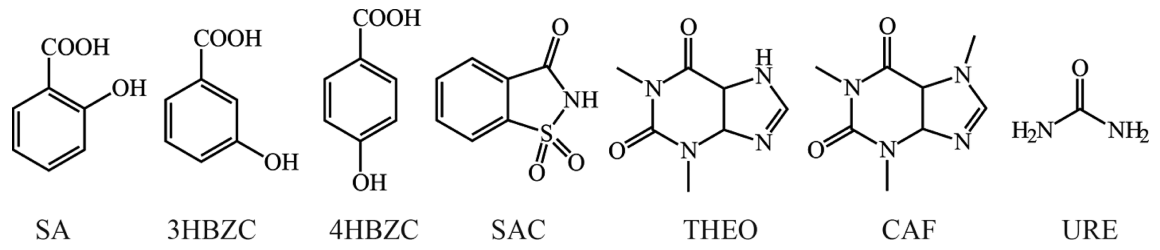

among possible crystalline modifications that can be used to improve the physicochemical and pharmaceutical properties of drugs. $^{4-10}$ Cocrystals have been successfully used in the pharmaceutical area to produce solid forms of a drug with improved properties. ${ }^{11-16}$ Caira and co-workers reported the crystal structure of several NVP cocrystals with improved dissolution rates compared to pure NVP crystals. ${ }^{17}$ So far, eutectic systems with NVP have not been described in the literature. However, for other drugs, pharmaceutical eutectic systems have shown an accomplishment in the production of solid forms with improved properties, including dissolution. ${ }^{8,18-20}$

Pharmaceutical cocrystals are formed with neutral molecules of an active pharmaceutical ingredient (API) and any other neutral molecule in a well-established stoichiometry. ${ }^{21,22}$ Cocrystals are single-phase compounds, exhibiting a new crystalline structure, which is different from that of parent components. Further, this new phase exhibits its own physicochemical properties, also different from the properties exhibited by parent components (Figure 1). In turn, physical mixtures and eutectic systems can be described as multiphase compounds. They exhibit a mixture of two or more phases that do not interact to form a new structure, such as in cocrystals (Figure 1). Physical mixtures exhibit the physicochemical properties of both parent compounds. However, at certain ratios, the eutectic mixture exhibits a lower melting point than the parent compounds and may also show differences in other physicochemical properties. ${ }^{10}$ Eutectic system formation occurs when the components are miscible in a liquid state and immiscible in the solid state. ${ }^{23}$ When two components, A and B, in the liquid phase are cooled, the solidification of both components and formation of a mixture of solid phases, $\alpha$ and $\beta$ occur. It can be concluded that an eutectic material has been obtained when it presents a single melting point which is dependent on the composition of the eutectic.

In order to produce multicomponent materials of NVP presenting better properties, a screening study was herein performed. The coformer molecules were selected based on the presence of carboxyl and amide groups that could disrupt the amide-amide dimer motif observed in the pure NVP structure, and that had delocalized planar structures that could stack with the pyridine fragments in NVP molecules. Thus, seven coformers, salicylic acid (SA) and its two isomers-3hydroxybenzoic acid (3HBZC), 4-hydroxybenzoic acid (4HBZC) - saccharin (SAC), caffeine (CAF), theophylline (THEO), and urea (URE) (Scheme 1), were selected and used in this screening of multicomponent forms of NVP. These materials were prepared through the liquid-assisted grinding (LAG) method, which is well-accepted for pharmaceutical cocrystal screening. ${ }^{24}$ Solid-state characterization was performed through single-crystal and powder X-ray diffraction with conventional and synchrotron radiation at different temperatures, differential scanning calorimetry, and solid-state nuclear magnetic resonance.

The crystal structure of NVP-SA and NVP-SAC cocrystals have been previously described by Caira and co-workers, ${ }^{17}$ and they were selected for reproducibility purposes and to compare with the $3 \mathrm{HBZC}$ and $4 \mathrm{HBZC}$ isomers behavior. Besides, a full 
characterization for both multicomponents, NVP-SA and NVP-SAC, has been done. NVP-SA crystals presented a temperature-dependent disorder. NVP-URE and NVP-4HBZC have been described by Nalte and co-workers. ${ }^{25}$ However, they have tested the cocrystal formation of these multicomponent forms only through melting point determination using an open capillary tube method. NVP-(3HBZC, 4HBZC, THEO, CAF, URE) multicomponent crystals structures were not found at the CSD database.

\section{EXPERIMENTAL SECTION}

Cocrystallization Prediction. In order to predict the propensity formation of NVP cocrystals, two Cambridge Crystallographic Data Centre (CCDC) tools were used: the screening by molecular complementarity $(\mathrm{MC})^{26}$ and the hydrogen-bond propensity (HBP). ${ }^{27}$ Both tools are available at CSD Mercury software version 4.0.0.

The MC is a tool developed and validated by L. Fábián, whose output is a simple pass or fail answer to the formation of the cocrystals. This is based on the premise that molecules tend to crystallize together only if they have similar molecular properties. Therefore, a few shape and polarity descriptors are calculated for the API and the coformer, and to pass the MC test, they have to differ by less than a threshold value that Fábián established from a statistical analysis performed at the CSD.

The HBP tool was originally developed as a knowledge-based method to assess the risk of polymorphism for a given compound, though it can also be used to evaluate cocrystal formation. On the basis of an automated statistical analysis of hydrogen bonding patterns, the HBP method determines interaction likelihoods for all the possible hydrogen-bonding interactions that can be formed with the set of functional groups present in the specific chemical environment analyzed. We have built three different mol2 files to analyze the seven cocrystal systems, one for each component (NVP and coformer) and another file containing both together. In this way, we can judge how likely a cocrystal is to form (multicomponent score) as the difference of the most likely interaction in pure NVP or in pure coformer and, the most likely cocrystal interaction. The HBP fitting data were generated using the truncate data generation mode.

Sample Preparation. The liquid-assisted grinding method was used to prepare the multicomponent materials containing NVP. Seven different coformers were tested: salicylic acid (SA), 3-hydroxybenzoic acid (3HBZC), 4-hydroxybenzoic acid (4HBZC), saccharin (SAC), caffeine (CAF), theophylline (THEO), and urea (URE). Experimental details are available in Supporting Information. Powder samples were characterized by solid-state analytical techniques.

Powder X-ray Diffraction (PXRD). Pure NVP and seven pure coformers were characterized by PXRD, which was carried out by using conventional (PXRD) and synchrotron (SPXRD) sources.

PXRD analyses were carried out in Rigaku automatic X-ray diffractometer for powder diffraction (Ultima IV) by using $\mathrm{Cu}-\mathrm{K} \alpha$ radiation source $(\lambda=1.5418 \AA)$. The $\mathrm{K} \beta$ radiation was filtered. Data were recorded at tube voltage of $40 \mathrm{kV}$ and a current of $30 \mathrm{~mA}$. Samples were placed on Bragg-Brentano (flat plate) geometry. ${ }^{28,29}$ The D/Tex Ultra detector operated at $2 \theta / \theta$ mode in continuous scanning at a scanning rate of $20 \% \mathrm{~min}$. Experiments were performed at room temperature, at step-size $0.01^{\circ}$ in the angular range $5-35^{\circ} 2 \theta$.

SPXRD experiments were performed at the XRD1 beamline at the Brazilian Synchrotron Light Laboratory (LNLS, Campinas, Brazil). ${ }^{30}$ This beamline is dedicated to X-ray powder diffraction analysis. It is composed of the three-circle heavy-duty diffractometer (Newport) and the MYTHEN 24K detector system (Dectris). Experiments were conducted in Debye-Scherrer geometry. Samples were placed in borosilicate capillaries $(0.7 \mathrm{~mm}$ diameter $)$. Experiments were conducted at $8 \mathrm{keV}$ radiation, and data were collected at the range 300 to $108 \mathrm{~K}$. Samples were cooled down using CryojetHT from OXFORD Instruments, at a cooling rate of $2 \mathrm{~K} \cdot \mathrm{min}^{-1}$. Equipment configuration allowed collecting one diffraction pattern every $1.2 \mathrm{~K}$.
Radiation wavelength was set based on Silicon standard (NIST SRM640D) data, which were collected at the end of each experiment.

Single-Crystal X-ray Diffraction (SXRD). SXRD experiments for NVP-SA were performed in a Bruker D8 Venture diffractometer (Photon $100 \mathrm{CMOS}$ detector and $\mathrm{MoK} \alpha$ radiation from Incoatec micro source) and for NVP-4HBZC in a Bruker D8 Venture diffractometer equipped with a CMOS Photon 100 detector using $\mathrm{CuK} \alpha$ radiation. The diffraction images were analyzed (indexed, integrated, and scaled) in the Apex 3 software. ${ }^{31}$ Crystal structures were solved through the direct methods and refined by Full-matrix-block least-squares in the SHELX-15 software. ${ }^{32}$ All non-hydrogen atoms were anisotropically refined, and all hydrogen atoms were placed in idealized geometries according to the riding model. Connectivity restraints and rigid body were used to describe salicylic acid molecule disorder at room temperature.

Differential Scanning Calorimetry (DSC). DSC curves were obtained in a DSC 204 F1 Phoenix NETZSCH calorimeter. In order to characterize the multicomponent materials, $5 \mathrm{mg}$ of each sample was placed in a hermetically sealed aluminum crucible and scanned at a temperature range of 50 to $300^{\circ} \mathrm{C}$, using a heating rate of $10^{\circ} \mathrm{C} \cdot \mathrm{min}^{-1}$. For the construction of phase diagrams, different compositions of eutectic systems were scanned at a temperature range of $50-300{ }^{\circ} \mathrm{C}$, using a heating rate of $3{ }^{\circ} \mathrm{C} \cdot \mathrm{min}^{-1}$. All samples were scanned in a nitrogen air atmosphere $\left(70 \mathrm{~mL} \cdot \mathrm{min}^{-1}\right)$, and an empty and sealed aluminum crucible was used as a reference. The equipment was calibrated by using indium ( $\mathrm{mp} 156.6^{\circ} \mathrm{C}$ and $\left.\mathrm{Hm} 28.54 \mathrm{~J} \cdot \mathrm{g}^{-1}\right)$ and zinc $\left(\mathrm{mp} 419.6^{\circ} \mathrm{C}\right)$. Data were processed in the NETZSCH Proteus software.

Solid-State NMR. ${ }^{13} \mathrm{C} \mathrm{CP} / \mathrm{MAS}$ ssNMR studies were performed using ramp CP/MAS pulse sequence ${ }^{33,34}$ with proton decoupling during acquisition at room temperature. All experiments were carried out in a Bruker Avance II spectrometer operating at a resonance frequency of $300.13 \mathrm{MHz}$ for protons and $75.46 \mathrm{MHz}$ for carbons. The spectrometer was equipped with a $4 \mathrm{~mm}$ MAS probe. The spinning rate was set at $10 \mathrm{kHz}$, the recycling delay was $350 \mathrm{~s}$, and the contact time during CP was $2 \mathrm{~ms}$. To obtain an adequate signal-to-noise ratio, 64192 transients were collected. The SPINAL- $64^{35}$ pulse sequence was used for heteronuclear decoupling during acquisition (40.96 ms) satisfying proton field $\mathrm{H} 1 \mathrm{H} \omega 1 \mathrm{H} / 2 \pi=\gamma \mathrm{H} \mathrm{H} 1 \mathrm{H} / 2 \pi=65.8 \mathrm{kHz}$. Glycine was used as an external reference $(\delta \mathrm{COOH}=176.46 \mathrm{ppm})$ and set to the Hartman-Hahn condition in CP/MAS experiments. Quaternary carbon edition spectra of all samples were recorded through a nonquaternary suppression (NQS) sequence; the ${ }^{1} \mathrm{H}$ and ${ }^{13} \mathrm{C}$ radiofrequency (rf) fields were removed during $40 \mu \mathrm{s}$ after $\mathrm{CP}$ and before the acquisition. Such delay allows carbon magnetization to decay because of ${ }^{1} \mathrm{H}-{ }^{13} \mathrm{C}$ dipolar coupling, which results in spectra wherein $\mathrm{CH}$ and $\mathrm{CH}_{2}$ are substantially removed. ${ }^{36}$

Dissolution Profile. Dissolution profiles were determined for NVP-THEO, NVP-CAF, and NVP raw material. The dissolution profiles were obtained in a Distek dissolution system Evolution 6100. It was used a USP apparatus II under stirring at $50 \mathrm{rpm}$. Experiments were carried in two different dissolution media: water and $\mathrm{HCl} 0.1 \mathrm{~N}$. A volume of $900 \mathrm{~mL}$ of the medium was placed in a vessel and maintained at $37 \pm 0.5{ }^{\circ} \mathrm{C}$ during all experiments. For each sample, $200 \mathrm{mg}$ of sample was dispersed in the medium. Experiments were performed in triplicate. An Opt-Diss 405 system (Distek), a multichannel, fiber opticbased UV spectrometer system, was attached to the dissolution system. It enabled us to collect the absorbance values and automatically calculated the percentage of dissolved material in each vessel. The system was set up to collect data every $30 \mathrm{~s}$ in the first 15 min, every $60 \mathrm{~s}$ in the following $45 \mathrm{~min}$, and every $10 \mathrm{~min}$ in the second hour.

Intrinsic Dissolution Rate. Intrinsic dissolution rate was calculated for NVP-THEO, NVP-CAF, and NVP raw material. A mass of $100 \mathrm{mg}$ of each sample was placed into the $0.8 \mathrm{~cm}$ diameter cavity in the apparatus. The powder was compressed under a pressure of $1600 \mathrm{psi}$ for $60 \mathrm{~s}$. The apparatus containing the compressed pellet was placed in $900 \mathrm{~mL}$ of medium. Two different media were used: water and $\mathrm{HCl} 0.1 \mathrm{~N}$. Previous experiments were performed to confirm that no phase transition occurred under pressure nor in the different media. 


\section{RESULTS AND DISCUSSION}

A liquid-assisted grinding method with NVP and seven different coformers (salicylic acid (SA), 3-hydroxybenzoic acid (3HBZC), 4-hydroxybenzoic acid (4HBZC), saccharin (SAC), caffeine (CAF), theophylline (THEO), and urea (URE)) was tested to obtain multicomponent materials. Four cocrystals confirmed by powder X-ray diffraction were obtained, NVP-SA, NVP-3HBZC, NVP-4HBZC, and NVP-SAC. Two eutectic materials, NVP-CAF and NVP-THEO, were confirmed and characterized through solid-state analytical techniques. The remaining one, NVP-URE, resulted in a solid physical mixture. The dissolution properties of the eutectic materials were investigated.

Cocrystallization Prediction Was Performed Using CCDC Tools. The molecular complementarity screening (MC) for the NVP API and the seven coformers was calculated (Table 1 and Table S1). Detailed information for all calculated

Table 1. Molecular Complementarity, MC, Results for Nevirapine Cocrystal Screening

\begin{tabular}{lc}
\hline Co-former & $\begin{array}{c}\text { Overall } \\
\text { PASS/FAIL }\end{array}$ \\
\hline Salicylic acid, SA & PASS/FAIL \\
3-Hydroxybenzoic acid, 3HBZC & PASS \\
4-Hydroxybenzoic acid, 4HBZC & PASS \\
Saccharin, SAC & PASS \\
Caffeine, CAF & PASS \\
Theophylline, THE & PASS \\
Urea, URE & FAIL \\
\hline
\end{tabular}

descriptor values is in Table S2. The results indicate that most of the selected coformers are likely to form NVP cocrystals. Urea is not expected to crystallize with NVP molecules. This is because the urea molecule has a fraction of $\mathrm{N}$ and $\mathrm{O}$ atoms overall nonhydrogen atoms present in the molecule, three times higher than NVP. While if SA is selected as a coformer, the propensity to crystallize with NVP depends on the SA conformer used from the CSD. Furthermore, the small $(S)$ axis of the imaginary calculated rectangular box that enclosed the URE molecule (MC method uses this box to define the shape and size descriptors) is much shorter than that calculated for NVP. None of these two descriptors pass the permitted MC threshold (Table S2). For the SA molecule, the difference in the S axis with NVP is close to the threshold, and a slight rotation of the hydrogens in the molecule modifies the length in the $S$ axis leading to pass or fail of the MC test.

The HBP analysis is available for polymorph assessment for one single molecule. Here, we have calculated HBP to assess the propensity of $\mathrm{H}$-bonding in a multicomponent formation, $\mathrm{AB}$, where $A$ is the NVP and B is the coformer. All hydrogen-bond donor and acceptor atoms of both molecules are considered.
The propensity is calculated for all donor $\cdots$ acceptor interactions between $\mathrm{A}-\mathrm{A}, \mathrm{B}-\mathrm{B}, \mathrm{A}-\mathrm{B}$, and $\mathrm{B}-\mathrm{A}$. It was considered the maximum propensity in each case (Table 2). A multicomponent score was calculated through the difference of the maximum propensity for heterointeractions, $\mathrm{A}-\mathrm{B}$ or $\mathrm{B}-\mathrm{A}$, which are probabilities (from 0 to 1 ) of each possible $\mathrm{H}$-bond, and the maximum propensity for $\mathrm{H}$-bond homointeractions, $\mathrm{A}-\mathrm{A}$ or $\mathrm{B}-$ B. Thus, a positive and higher multicomponent score means a greater propensity to form heterointeractions, and consequently, a higher probability to obtain multicomponent structures. Considering the nature of cocrystal and eutectic structures, heterointeractions are observed in cocrystals and in the interdomain surface in eutectics; therefore, high positive multicomponent scores must be related to cocrystal prediction. We should note that organic eutectics are conglomerates of lattice structures of the components where heterointeractions only appear in the interphase between domains.

The multicomponent scores obtained for the NVP cocrystals with the seven coformers are shown in Table 2 using a traffic light analogy. SA, 3HBZC, and 4HBZC, in green, are the most likely molecules to form NVP multicomponent solids. It agrees with our experimental results. On the other side, CAF exhibits the highest negative score. This molecule does not have any $\mathrm{H}$ bond donors, which affects the propensity results. Finally, SAC, THEO, and URE, in yellow, exhibit intermediate multicomponent score values. Only one-phase multicomponent materials should be predicted by the HBP tool for SAC, THEO, and URE because only in these solids H-bond interactions between the components are expected.

Powder X-ray Diffraction (PXRD). Analysis was carried out in order to identify the crystalline phases obtained after sample preparation. Diffraction patterns of all prepared samples were compared to diffraction patterns of its parental compounds. PXRD results indicate that NVP-SA, NVP3HBZC, NVP-4HBZC, and NVP-SAC samples correspond to new phases, whereas NVP-THEO, NVP-CAF, and NVPURE are a mixture of NVP and coformer phases (Figure S1). Furthermore, NVP-SA and NVP-SAC PXRD data were also compared to structures reported at the CSD. Comparing NVPSAC with the reported structure (CSD refcode: LATQOO), ${ }^{17}$ one can conclude that we have obtained the same crystallographic phase recorded by Caira et al. (Figure S2). However, a comparison of NVP-SA and the reported cocrystal (CSD refcode: LATQUU) ${ }^{17}$ evidence some differences. It is important to mention that PXRD data were collected at room temperature (RT) and Caira's reported structure was determined at low temperature $(100 \mathrm{~K}) .{ }^{17}$ Significant differences were observed at approximately $12.5^{\circ}$ and in the region between $15^{\circ}$ and $20^{\circ}$, which could be evidence of structural changes with the temperature (Figure S3). Further characterization was per-

Table 2. HBP Results for Multicomponent Analysis ${ }^{a}$

\begin{tabular}{lccccc}
\hline \multicolumn{1}{c}{ Component B } & $\begin{array}{c}\text { Max } \\
\text { interaction }\end{array}$ & $\begin{array}{c}\text { Max A:B or } \\
\text { B:A propensity }\end{array}$ & $\begin{array}{c}\text { Max A:A } \\
\text { propensity }\end{array}$ & $\begin{array}{c}\text { Max B:B } \\
\text { propensity }\end{array}$ & $\begin{array}{c}\text { Multicomponent } \\
\text { score }\end{array}$ \\
\hline Salicylic acid, SA & B:A & 0.69 & 0.52 & 0.33 & 0.17 \\
3-Hydroxybenzoic acid, 3HBZC & B:A & 0.69 & 0.52 & 0.34 & 0.17 \\
4-Hydroxybenzoic acid, 4HBZC & B:A & 0.70 & 0.53 & 0.34 & 0.17 \\
Theophylline, THE & B:A & 0.63 & 0.44 & 0.56 & 0.07 \\
Urea, URE & B:B & 0.94 & 0.45 & 0.95 & -0.01 \\
Saccharin, SAC & B:B & 0.50 & 0.42 & 0.57 & -0.07 \\
Caffeine, CAF & A:A & 0.25 & 0.41 & -- & -0.16 \\
\hline
\end{tabular}

${ }^{a}$ Component A refers to the NVP molecule. 

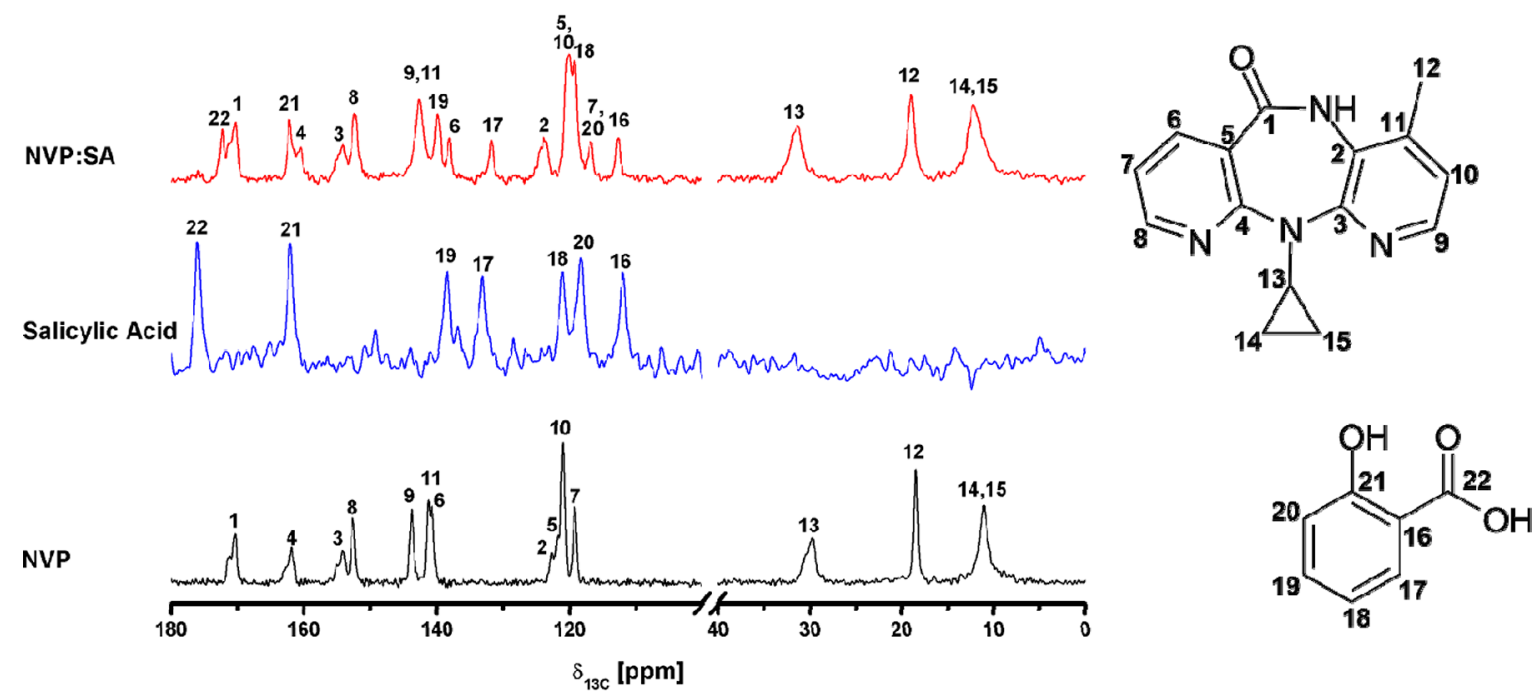

Figure $2 .{ }^{13} \mathrm{C} C P$ /MAS spectra of NVP-SA, SA, and NVP. Carbon numbering adopted throughout the study is highlighted.

formed in order to understand these differences in the diffraction patterns, and the results will be presented later.

Solid-State NMR. Analysis was carried out in NVP-SA, NVP-SAC, NVP-THEO, NVP-CAF, and NVP-URE samples. The ${ }^{13} \mathrm{C} \mathrm{CP} /$ MAS spectra of NVP and SA are shown in Figure 2. The carbon assignments and chemical shift values in all spectra are given in Table S3. The assignments were done considering the NQS spectra. The ${ }^{13} \mathrm{C} \mathrm{CP} / \mathrm{MAS}$ spectrum did not show multiplicity in the resonance lines in both cases, thus indicating only one molecule per asymmetric unit. The ${ }^{13} \mathrm{C} \mathrm{CP} /$ MAS spectrum of NVP-SA is also shown in Figure 2. Clear changes in the chemical shifts of signals can be found by comparing NVP-SA with the coformers. Thus, it is possible to ensure the existence of an interaction between NVP and SA.

The ${ }^{13} \mathrm{C} \mathrm{CP} /$ MAS spectra of the NVP-SAC sample exhibit changes in the chemical shift of the signals regarding the spectra of the precursors. This is an indication of modifications in the environments of both molecules and the presence of interactions between them (Figure S4). On the other hand, the ssNMR ${ }^{13} \mathrm{C}$ spectra for NVP-THEO, NVP-CAF, and NVP-URE are the addition of the spectra of NVP and the respective coformer (Figure S4). This result evidences that there is no interaction between the pure compounds; i.e., the result of the cocrystallization process is the physical mixture of the precursors. These results agree with that obtained by PXRD.

Differential Scanning Calorimetry (DSC). Analysis was carried out in all multicomponent samples. As expected, NVPSA, NVP-3HBZC, NVP-4HBZC, and NVP-SAC cocrystals were also confirmed through DSC. Although Nalte and coworkers $^{25}$ have described NVP-URE as a cocrystal, with a different melting point, the DSC results indicate that this sample consists of a physical mixture. In the cases of NVP-THEO and $\mathrm{NVP}-\mathrm{CAF}$, interesting thermal behavior was noticed and is commented on in the sequence. All curves are available in the Supporting Information (Figure S5).

According to PXRD and ssNMR analysis, one can conclude that the NVP-THEO and NVP-CAF are simply physical mixtures of NVP and coformer. However, DSC results showed that NVP-THEO and NVP-CAF behavior is not that expected for physical mixtures. In the case of the NVP-THEO, the multicomponent system presented two thermal events at 223.6 and $234.1^{\circ} \mathrm{C}$; however, NVP melts at $247.8^{\circ} \mathrm{C}$, whereas THEO melts at $274.7{ }^{\circ} \mathrm{C}$. Since the mixture melts in a temperature below the melting point of the pure compounds, the data exhibited for NVP-THEO would allow thinking that this could be a eutectic system. NVP-CAF presented a similar behavior. The DSC analysis for the NVP-CAF sample showed thermal events at $163.1{ }^{\circ} \mathrm{C}, 204.4{ }^{\circ} \mathrm{C}$, and $212.8{ }^{\circ} \mathrm{C}$. The pure $\mathrm{CAF}$ presented two main events at 155.8 and $238.6^{\circ} \mathrm{C}$. The first point was consistent with data in the literature, which indicates a phase transition for caffeine at $153{ }^{\circ} \mathrm{C},{ }^{37}$ whereas the second event corresponds to the melting point of CAF. Thus, the event occurring at $163.1{ }^{\circ} \mathrm{C}$ corresponds to the phase transition of $\mathrm{CAF}$; however, the following two events are not related to pure CAF or to pure NVP. Adding this evidence to PXRD and ssNMR results, one can conclude that NVP-CAF could also be a eutectic system.

In order to investigate the eutectic systems, different compositions of NVP-THEO and NVP-CAF were analyzed through DSC. Eleven curves were obtained for each system (Figure S6), corresponding to pure NVP, pure coformer (THEO or CAF), and samples at ratios of 1:9, 2:8, 3:7, 4:6, $5: 5,6: 4,7: 3,8: 2$, and $9: 1(\mathrm{~m} / \mathrm{m})$. Samples were prepared by a simple mixture of components in the absence of solvent. Both systems, NVP-THEO and NVP-CAF, present similar behaviors. For each set of a mixture, there is a curve where only one event appears, and it corresponds to the eutectic composition of the system. In all the other curves, there is an event corresponding to the melt of the eutectic, followed by a second event. This second event corresponds to the excess of NVP or coformer, which has a variable melting point according to the composition. In the case of NVP-THEO, the eutectic composition occurs in a ratio of $7: 3(\mathrm{~m} / \mathrm{m})$, presenting an eutectic temperature of $224.1^{\circ} \mathrm{C}$ approximately. In the ratios of $8: 2$ and $9: 1$, the second event corresponds to the melting of NVP, whereas in the ratios of $1: 9,2: 8,3: 7,4: 6,5: 5$, and $6: 4$, it corresponds to the melting point of THEO (Figure S6a). For the NVP-CAF system, the eutectic composition occurs in a ratio of $3: 7(\mathrm{~m} / \mathrm{m})$, with a eutectic temperature of $203.4{ }^{\circ} \mathrm{C}$ approximately. In ratios of $1: 9$ and $2: 8$, the second event corresponds to the melting point of CAF, whereas in the ratios of $4: 6,5: 5,6: 4,7: 3,8: 2$, and 9:1, it corresponds to NVP (Figure S6b). 

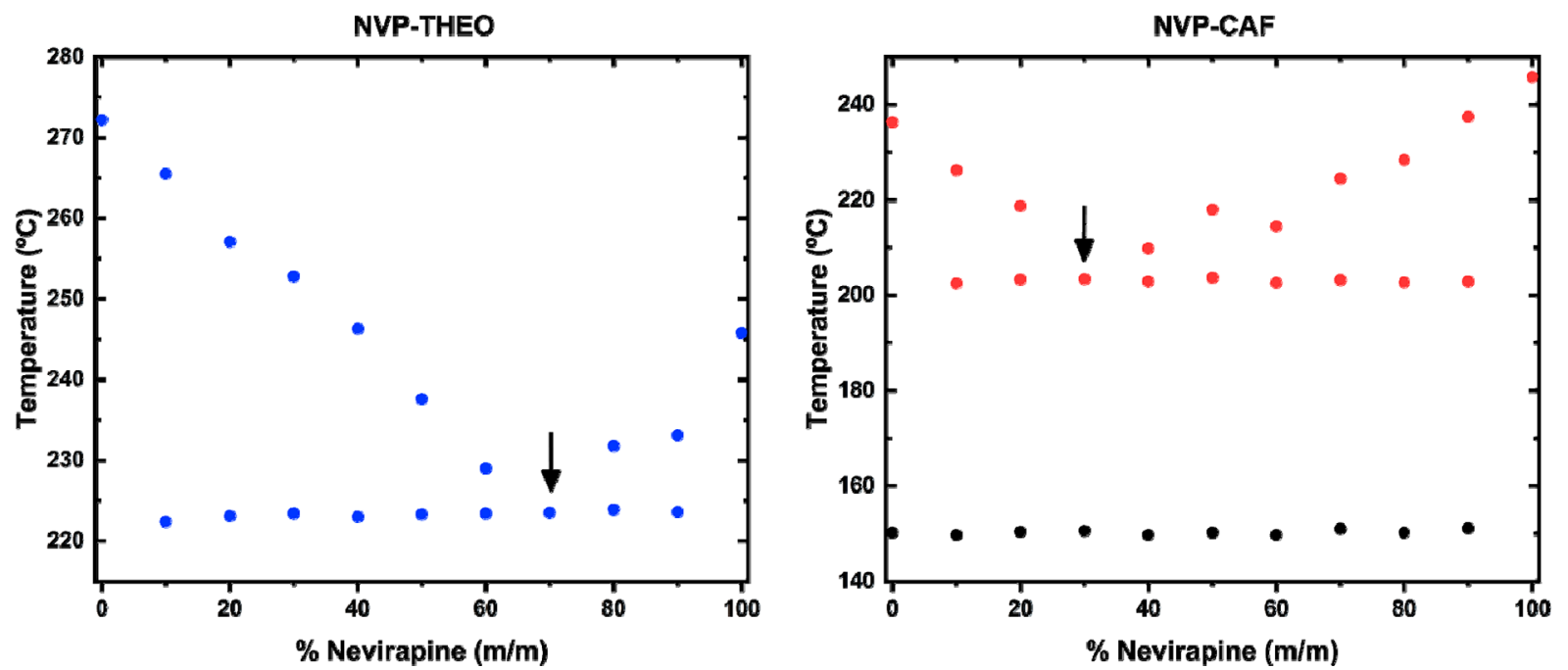

Figure 3. Phase diagrams for NVP-THEO and NVP-CAF systems. The arrows indicate the eutectic points in each system. In the NVP-CAF phase diagram, besides the liquidus and solidus lines, it is also possible to see the caffeine phase transition (black dots).

On the basis of the thermal curves obtained at different compositions, phase diagrams for both systems were obtained (Figure 3). In the case of NVP-THEO, one can clearly distinguish the liquidus and solidus lines. The solidus line corresponds to the constant curve at approximately $222^{\circ} \mathrm{C}$. This line marks the temperature where the eutectic mixture starts to melt, that is, the eutectic temperature. In the case of NVP-CAF, an eutectic temperature around $\sim 202{ }^{\circ} \mathrm{C}$ is present in all compositions, marking the solidus line for this diagram, and consequently, the eutectic temperature. In addition, a line around $153{ }^{\circ} \mathrm{C}$ can be seen and corresponds to the phase transition of CAF. The intersection of the liquidus lines and the solidus line in each diagram allows us to obtain the eutectic composition in each system. Using the linear fitting, it was possible to determine the eutectic composition of 70:30 (\% m/ $\mathrm{m}$ ) for NVP-THEO and 36:64 (\% $\mathrm{m} / \mathrm{m})$ for NVP-CAF.

Single Crystal X-ray Diffraction. In order to determine the crystalline structure of the four cocrystals obtained, slow evaporation experiments were carried out for all of them attempting to obtain good single crystals for SCXRD experiments. Although single crystals were obtained for NVP-SA and NVP-4HBZC, so far it has not been possible to grow crystals of suitable quality to carry out SCXRD experiments for NVP-SAC and NVP-3HBZC. Crystallographic parameters are summarized in Table S4. Structures of the NVP-SA multicomponent at room temperature (RT) and $100 \mathrm{~K}(\mathrm{LT})$ present the same space group, $P \overline{1}$; however, the second has twice the volume than the first, and while the NVP-SA at RT has $z^{\prime}$ equal to 1 , the LT structure has $z^{\prime}$ equal to 2 . Both present a stoichiometry NVP coformer 2:1. Salicylic acid molecules at RT are positioned in a center of symmetry that confers disorder to them (Figure 4). The precession images (Figure S7) verify the confidence of the assigned unit cell in both cases, since the unit cell at room temperature could not explain all the observed reflections in the experiment at low temperature and corroborates the loss of the inversion center of symmetry at the NVP dimer and over the salicylic acid molecules (Figure 5 and Figure 6), which doubles the asymmetric unit and, therefore the unit cell volume (Table S5). NVP-4HBZC crystallizes in the $C 2 / c$ monoclinic space group, and it presents one nevirapine molecule and one 4hydroxybenzoic acid molecule in the asymmetric unit (Figure 7). Nevirapine molecules in the title structures do not present

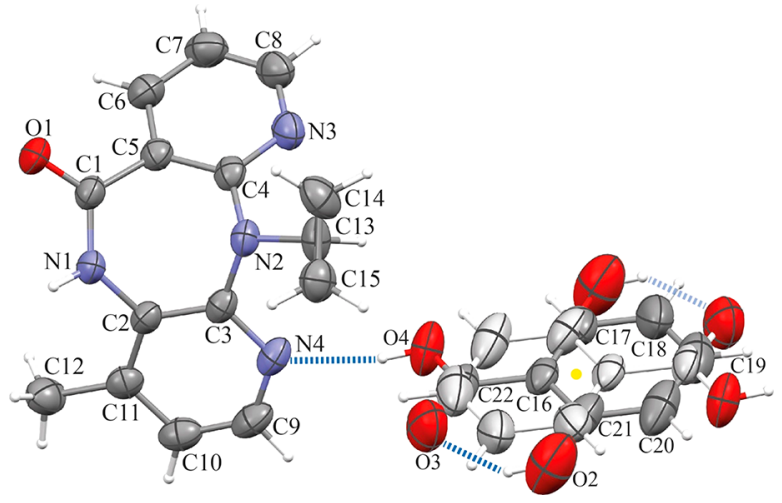

Figure 4. Representation of the NVP-SA cocrystal asymmetric unit at room temperature (inversion center indicated by a yellow dot and hydrogen bond interactions as dashed blue lines). Thermal ellipsoids drew at the $50 \%$ probability level.

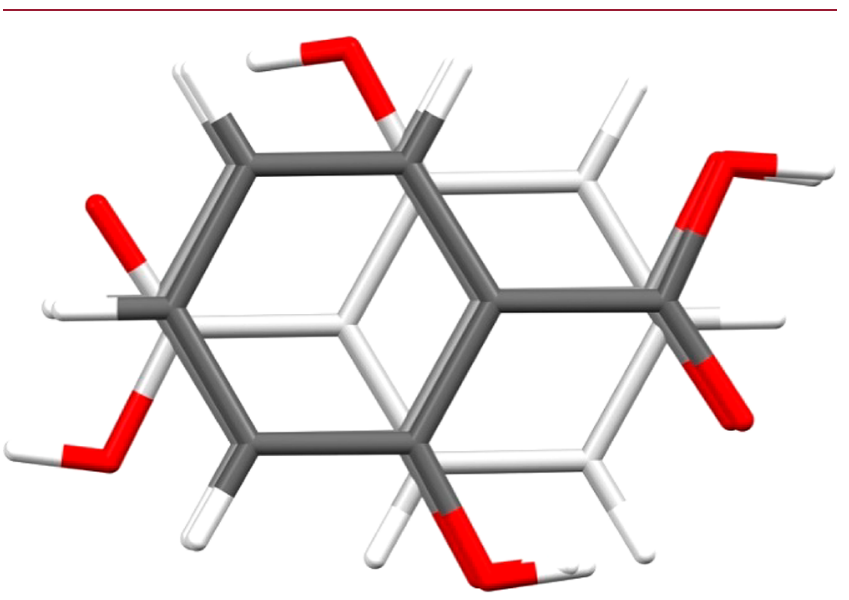

Figure 5. Comparison of the SA molecule position in the NVP-SA structures at room temperature (white) and at $100 \mathrm{~K}$ (gray).

significant differences in bond distances and angles. They display a "butterfly" conformation with angles between the pyridine rings at the range $\left(119.9-126.4^{\circ}\right)$ in agreement with pure NVP structure (CSD refcode: PABHIJ01) ${ }^{38} 121.9^{\circ}$. 


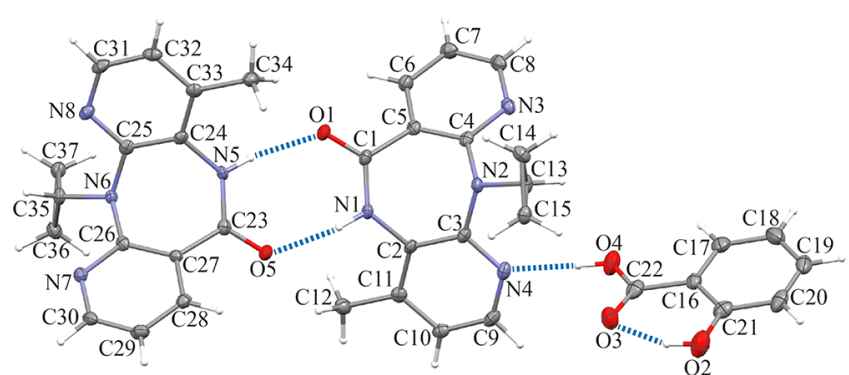

Figure 6. Representation of the NVP-SA cocrystal asymmetric unit at $100 \mathrm{~K}$ (hydrogen bond interactions depicted as dashed blue lines). Thermal ellipsoids drawn at the $50 \%$ probability level.

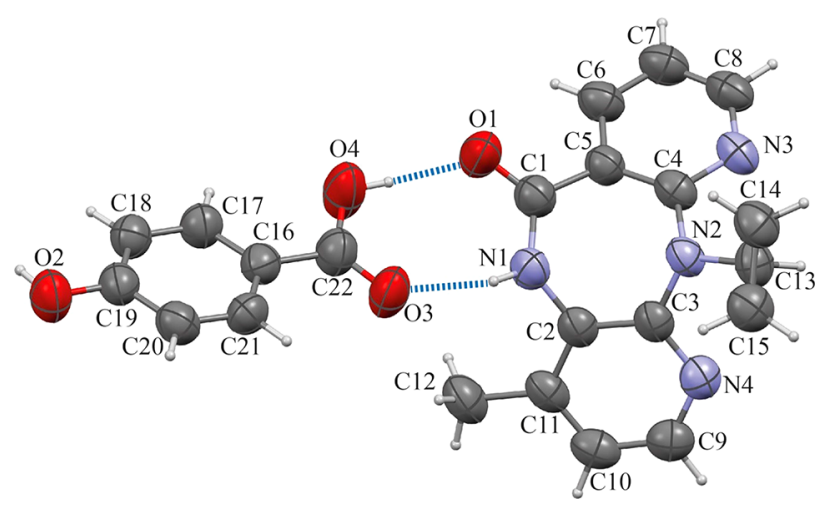

Figure 7. Representation of the NVP-4HBZC cocrystal asymmetric unit (hydrogen bond interactions depicted as dashed blue lines). Thermal ellipsoids drawn at the $50 \%$ probability level.

Nevirapine molecules in the NVP-SA structure form homodimers through amide-amide interactions that display a motif with graph set R2,2(8). In NVP-4HBZC, a hydrogen motif with the same graph set R2,2(8) is also observed, but it corresponds to a hydrogen bond motif formed between the carboxylic acid in 4HBZC and the amide group in NVP, displaying heterodimers.

If we compare the crystal packing that displays pure nevirapine in PAHBIJ01 ${ }^{38}$ with the nevirapine molecules packing in the NVP-SA and NVP-4HBZC cocrystal structures, the observed infinite pyridine stacking $(\pi \cdots \pi$ interactions) of nevirapine molecules observed in PABHIJ01 is also conserved in NVP-SA and NVP-4HBZC cocrystals (Figure 8). Furthermore, if we consider in the strong amideamide hydrogen bonds, these chains grow into mimic layers for PABHIJ01 and NVP-SA (Figure 8d). NVP-4HBZC does not present homodimers and therefore does not form these layers. However, the cocrystal reported by Caira et al. of NVP and SAC (LATQOO $)^{17}$ also presents mimic 1D infinite chains and 2D layers in its crystal structure. The way in which these layers are packed in the crystal is different, while in the PABHIJ01 structure there are no holes; in NVP-SA and NVP-SAC (LATQOO) cocrystals, the layers pack forming parallel pipes that allow the SA and SAC molecules to be located forming tapes (Figure 9 and Figure S8).

Justified by the fact that SA molecules are situated in pipeshaped channels along with the NVP cocrystal structure, we propose a dynamic disorder at the RT structure. Salicylic acid molecules rotate $180^{\circ}$ synchronously along with the crystal probably due to the low energy barrier between both conformations, while, SA molecules are not able to present dynamic effects in the crystal at $100 \mathrm{~K}$. This disorder can also explain the Caira et al. structure recorded at the CSD with LATQUU refcode, ${ }^{17}$ which presents the same unit cell and space group as our structure at low temperature. Both data were collected at $100 \mathrm{~K}$; however, the experiments were carried out in a different manner. We kept the crystal mounted at the goniometer head during the cooling ramp, while in LATQUU, the crystal was frozen instantly. This can obviously make a difference; a ramped temperature allows a conformational selection, while fast freezing does not. In addition, rapid freezing produces a disorder in the salicylic acid molecule, showing two orientations: the major, presenting a final refined site occupancy factor of 0.74 , coinciding with the observed in our LT structure; and the minor, with occupancy of 0.26 , which is suggested as a possible "intermediate state" (Figure 9).

Powder Dissolution Profile and Intrinsic Dissolution Rate (IDR). As the cocrystals, eutectic systems can present advantages over the pure drug. In order to evaluate the impact of
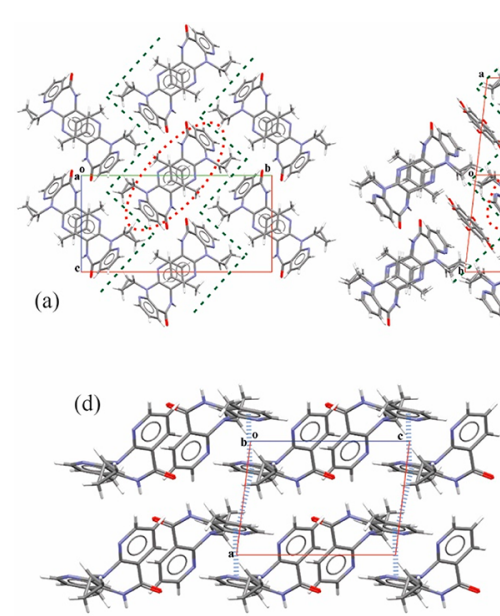
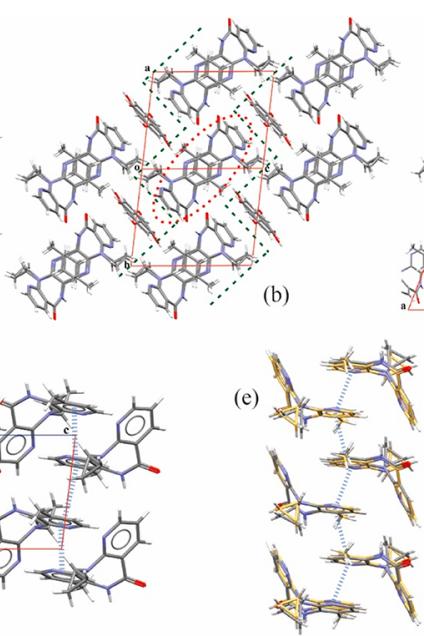
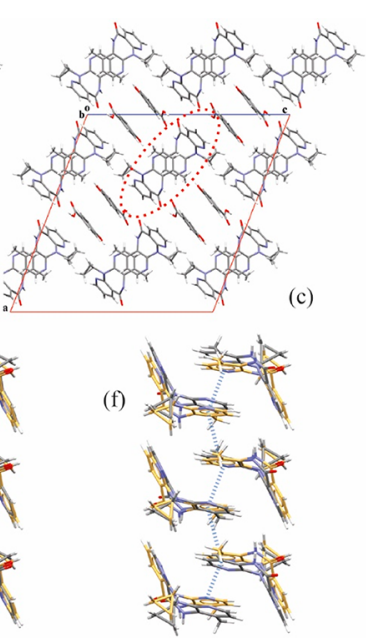

Figure 8. Crystal structure of (a) pure nevirapine, PABHIJ01, (b) NVP-SA at $100 \mathrm{~K}$ and (c) NVP-4HBZC. Red dotted ellipses round infinite NVP chains and zigzag dashed green lines frame the nevirapine layers that are common into PABHIJ01 and NVP-SA packing. (d) Perpendicular projection of a nevirapine layer for the PABHI01 compound. Superposition of nevirapine chains for the structures: (e) PABHIJ01 (yellow) and NVP-SA (gray), (f) PABHIJ01 (yellow) and 4HBZC (gray). 

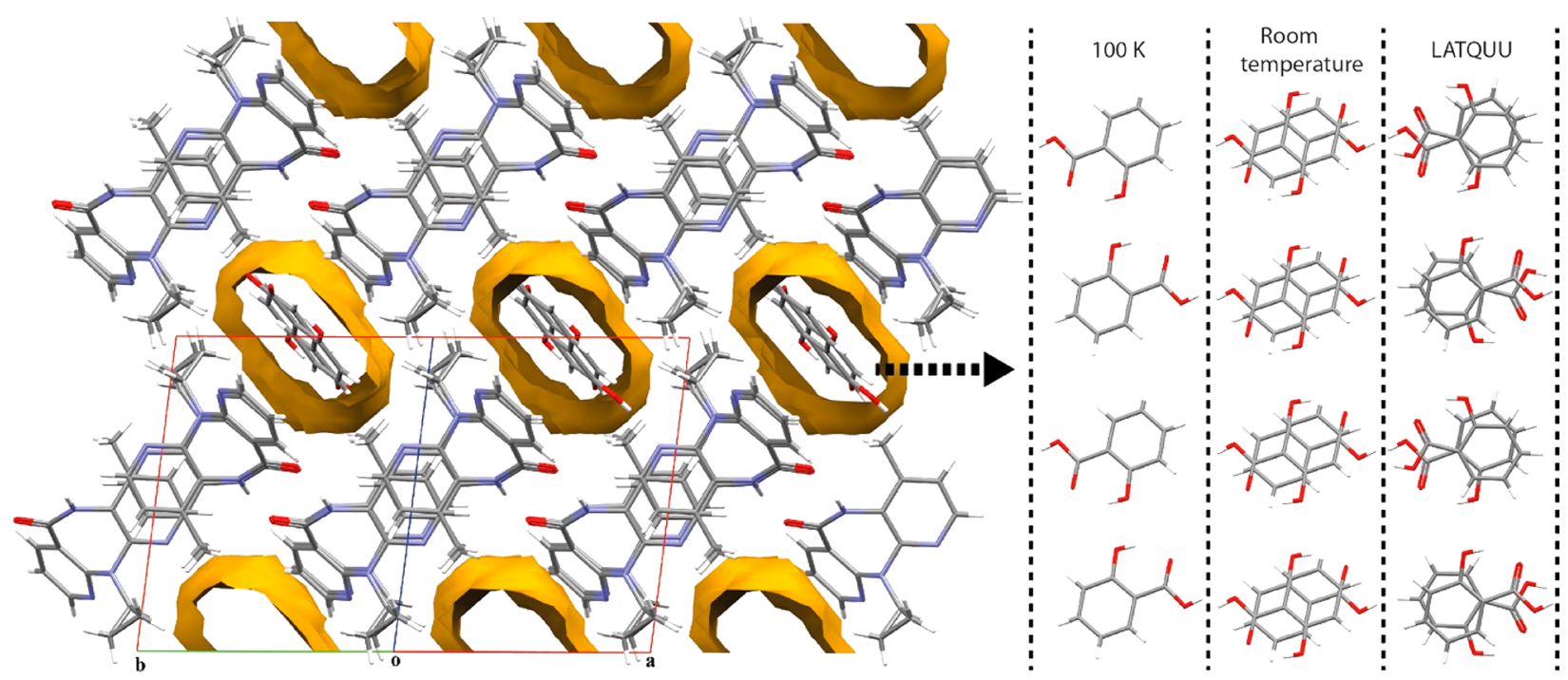

Figure 9. Structure of NVP-SA showing the pipe-shaped voids in yellow and on the right, the extended projection of the observed SA tapes that fit in those channels for the three discussed NVP-SA cocrystals.
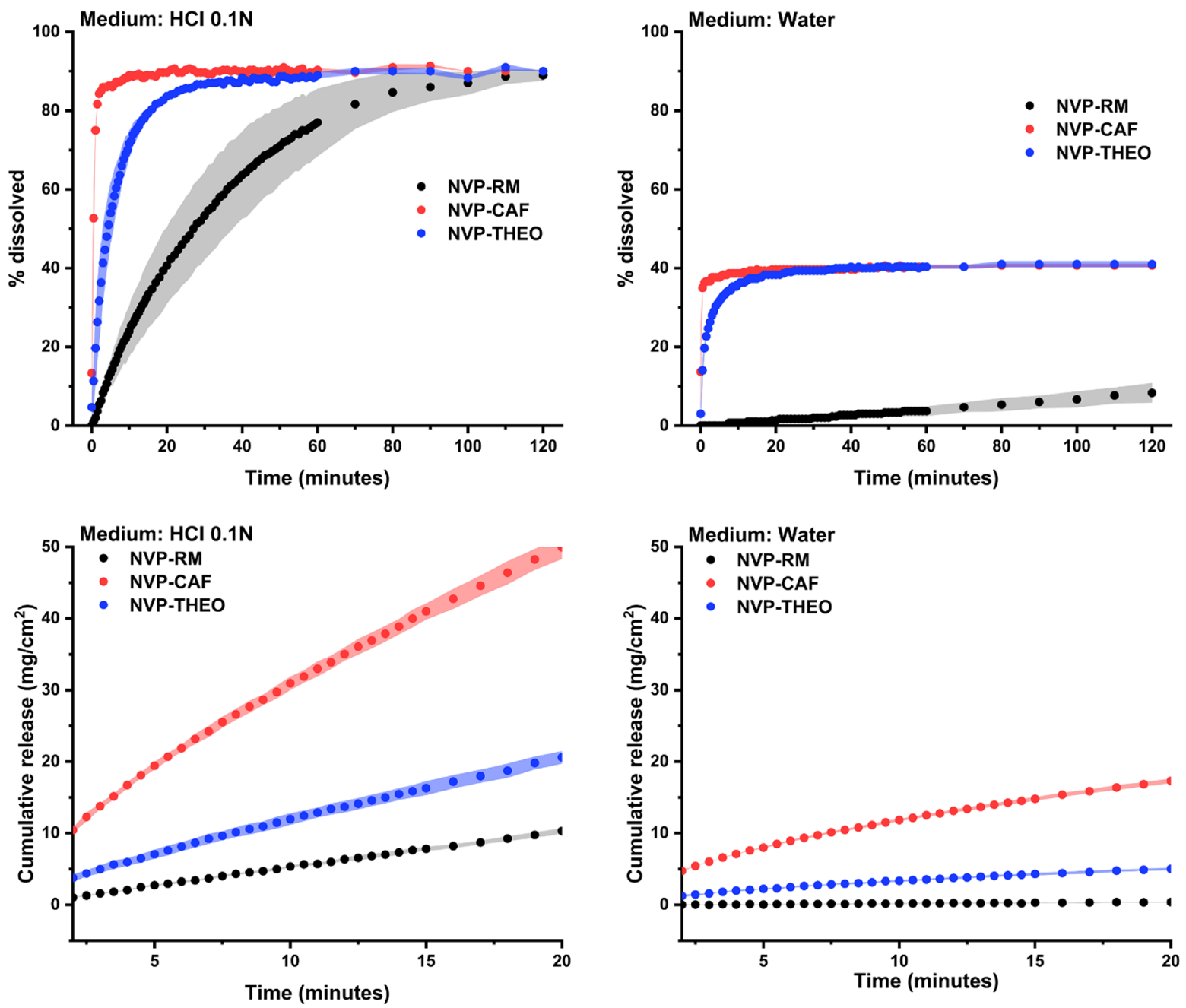

Figure 10. Dissolution profile of NVP in eutectic systems in comparison to pure NVP at $0.1 \mathrm{~N} \mathrm{HCl}$ and water. Intrinsic dissolution rates of NVP in the same samples were also determined for the same dissolution media.

eutectic systems in the dissolution properties, the powder dissolution profiles and the intrinsic dissolution rates of pure
NVP, NVP-THEO, and NVP-CAF in water and $0.1 \mathrm{~N} \mathrm{HCl}$ medium were determined (Figure 10). 
In the $0.1 \mathrm{~N} \mathrm{HCl}$ medium, while pure NVP takes more than $80 \mathrm{~min}$ to dissolve $80 \%$ of its initial amount, NVP-THEO takes less than $20 \mathrm{~min}$ and NVP-CAF takes less than $5 \mathrm{~min}$ to dissolve the same amount. These results clearly show that the eutectics exhibit an advantage with respect to the pure NVP relative to the dissolution kinetics. In water, this advantage is even greater. While less than $5 \%$ of pure NVP dissolves over $2 \mathrm{~h}$, eutectic materials reach $40 \%$ of dissolved material in less than 20 min for NVP-THEO and in less than 5 min for NVP-CAF.

Although IDR was considered a parameter relevant for the biopharmaceutical classification system in the past, ${ }^{39,40}$ the literature indicates that nowadays it is been more relevant as a solid-state characterization technique. ${ }^{41,42}$ As previously described, all the samples show different crystal structures, and so the intrinsic dissolution behavior was expected to be different too. All the samples presented $R^{2}$ values higher than 0.9 , showing that no transition occurred during the test. Moreover, just dissolution values of less than $10 \%$ were considered to plot the results. Both modifications, with THEO and CAF, showed IDR higher than that of the pure raw material.

So, both dissolution tests proposed here can be used to discriminate between the raw material and the prepared samples and also between these two modifications made. It can be concluded that the modifications were successful in the generation of higher dissolution rate structures.

\section{CONCLUSIONS}

In the attempt to improve the physicochemical properties of the antiretroviral nevirapine, we have chosen seven compounds to search for NVP multicomponents. They have been chosen because of their possible ability to alter the interactions observed in the packing arrangement of pure NVP because these molecules contain carboxylic acid, amide, and planar electrondelocalized fragments in their formula. The multicomponent samples were characterized through solid-state techniques, and the results indicate that our initial criterium was quite right, and four cocrystals (NVP-SA, NVP-SAC, NVP-3HBZC, NVP4HBZC), two eutectics (NVP-THEO, NVP-CAF), and one (NVP-URE) physical mixture were identified. Moreover, we have used the multicomponent prediction tools available in the CSD to confirm whether they can be helpful in this type of study. Results have shown they are adequate. Molecular complementarity (MC) tool establishes all coformers as suitable to form cocrystals or multicomponents with NVP except for urea. With the hydrogen bond propensity (HBP) tool, a high probability of obtaining cocrystals for SA, 3HBZC, and $4 \mathrm{HBZC}$ is predicted; for SAC, THEO, and URE the probabilities are almost nil, and, in the case of CAF, the results show incompatibility to form cocrystals with NVP.

Even though the methodology used to achieve the multicomponent structures is different from those used by Caira (reference), the same crystalline phase was obtained for NVP$\mathrm{SA}$ and NVP-SAC as the one reported in the literature. Moreover, the NVP-SA structures obtained in this work and their comparison with LATQUU allowed us to identify a temperature-dependent dynamic disorder behavior of the salicylic acid molecules along the channels displayed in the NVP host crystal. Further studies are required, and they will be presented in a future manuscript.

NVP-THEO and NVP-CAF results comply with the formation of eutectics since two different phases and a single melting point were determined. In order to start understanding the behavior of these two systems, phase diagrams were obtained. Also, the composition and melting point of these eutectics were determined. Moreover, dissolution studies have demonstrated an improvement in the dissolution kinetics behavior of these materials compared to pure NVP, especially in the aqueous medium.

Finally, once again multicomponent materials are presented as a good strategy to improve the properties of pharmaceutical drugs, and knowledge-based methods are useful tools for selecting molecules with the highest probability of crystallizing with an API.

\section{DEDICATION TO JOEL BERNSTEIN}

This paper is dedicated to Joel Bernstein, and the reason is the following. Joel was a great inspiration everywhere and, mainly, in Latin America. This paper is a collaboration between students and researchers of both Latin American countries, Brazil and Argentina. Joel was visiting and exchanging ideas and knowledge with us in Brazil and Argentina for three months during 2013. We invited him for different events and lab visits in Brazil (Workshop of Polymorphism and Nanotechnology of Pharmaceutical Drugs and International School of Crystallography and Crystallization - II ECRISLA). After that, he went to Argentina and participated in the First Latin American Meeting of Crystallography in Córdoba. The Latin American Crystallography Association (LACA) was created in that opportunity.

For all of that, we will be eternally grateful to Joel. Finally, with great pleasure, we share with you Joel's dedication to a wonderful book in which he expressed his feelings about his first visit to Latin America (Figure 11).

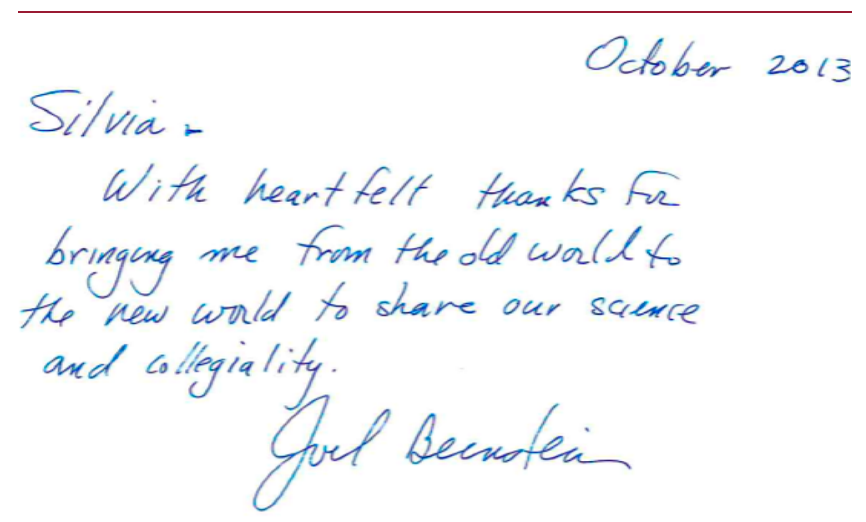

Figure 11. Note written by Joel Bernstein after his first visit to Latin America, Brazil, and Argentina.

\section{ASSOCIATED CONTENT}

\section{Supporting Information}

The Supporting Information is available free of charge at https://pubs.acs.org/doi/10.1021/acs.cgd.9b01129.

Experimental details, prediction detailed results (Tables S1-S2), PXRD data (Figures S1-S3, S9), solid-state NMR data (Table S3 and Figure S4), DSC curves (Figures S5-S6), a summary of the crystallographic data (Tables S4-S5 and Figures S7-S8), and additional discussion (PDF)

\section{Accession Codes}

CCDC 1941056-1941057 and 1964383 contain the supplementary crystallographic data for this paper. These data can be obtained free of charge via www.ccdc.cam.ac.uk/data_request/ 
cif, or by emailing data_request@ccdc.cam.ac.uk, or by contacting The Cambridge Crystallographic Data Centre, 12 Union Road, Cambridge CB2 1EZ, UK; fax: +44 1223336033.

\section{AUTHOR INFORMATION}

\section{Corresponding Authors}

*(R.N.C) E-mail: rogeria.ncosta@gmail.com.

*(S.L.C) E-mail: scuffini@gmail.com.

\section{ORCID}

Rogeria N. Costa: 0000-0002-2410-0941

Ana L. Reviglio: 0000-0003-2359-6479

Gustavo A. Monti: 0000-0001-6531-798X

Alexandre M. G. Carvalho: 0000-0001-6439-414X

Duane Choquesillo-Lazarte: 0000-0002-7077-8972

\section{Present Address}

\#(A.M.G.C.) Centro de Tecnologia, Departamento de Engenharia Mecânica, Universidade Estadual de Maringá (UEM), 87020-900 Maringá, Brazil.

\section{Notes}

The authors declare no competing financial interest.

\section{ACKNOWLEDGMENTS}

This study was financed in part by the Coordenação de Aperfeiçoamento de Pessoal de Nível Superior - Brasil (CAPES) - Finance Code 001. R.N.C. thanks CAPES for the Ph.D. scholarship. Part of this work was conducted during a scholarship supported by the International Cooperation Program CAPES/MINCyT. The study was also financed by CONICET Argentina Grant 11220130100746CO, ANPCYT Argentina Grants PICT 1095/2014, SeCyT UNC Argentina. A.L.R. thanks CONICET for the Ph.D. scholarship. J.A.L.C.R. thanks $\mathrm{CNPq}$ for Grant No. 311142/2017-6. L.I., S.L.C., and R.N.C. acknowledge Consejo Superior de Investigaciones Científicas for funding Project ICOOPA2015 COOPA20094. D.C.-L. acknowledges Ministerio de Ciencia, Innovación y Universidades of Spain for funding Project PGC2018-102047-B-I00. The authors acknowledge the donation of nevirapine raw material by Fundação Oswaldo Cruz (FIOCRUZ). R.N.C. would also like to thank Dra Gabriela Rauber for all the important discussions and for helping in the development of this work.

\section{REFERENCES}

(1) Hargrave, K. D.; Proudfoot, J. R.; Adams, J.; Grozinger, K. G.; Schmidt, G.; Engel, W.; Trummlitz, G.; Eberlein, W. 5,11-Dihydro-6HDipyrido(3,2:B-2',3'-e)(1,3)Diazepines and Their Use in the Prevention or Treatment of HIV Infection. US005620974A, 1994.

(2) Mui, P. W.; Jacober, S. P.; Hargrave, K. D.; Adams, J. Crystal Structure of Nevirapine, a Non-Nucleoside Inhibitor of HIV-1 Reverse Transcriptase, and Computational Alignment with a Structurally Diverse Inhibitor. J. Med. Chem. 1992, 35, 201-202.

(3) Lindenberg, M.; Kopp, S.; Dressman, J. B. Classification of Orally Administered Drugs on the World Health Organization Model List of Essential Medicines According to the Biopharmaceutics Classification System. Eur. J. Pharm. Biopharm. 2004, 58 (2), 265-278.

(4) Rodríguez-Hornedo, N.; Nehm, S. J.; Seefeldt, K. F.; PagánTorres, Y.; Falkiewicz, C. J. Reaction Crystallization of Pharmaceutical Molecular Complexes. Mol. Pharmaceutics 2006, 3 (3), 362-367.

(5) Remenar, J. F.; Morissette, S. L.; Peterson, M. L.; Moulton, B.; MacPhee, J. M.; Guzmán, H. R.; Almarsson, Ö. Crystal Engineering of Novel Cocrystals of a Triazole Drug with 1,4-Dicarboxylic Acids. J. Am. Chem. Soc. 2003, 125 (28), 8456-8457.
(6) Reddy, L. S.; Bethune, S. J.; Kampf, J. W.; Rodríguez-Hornedo, N. Cocrystals and Salts of Gabapentin: PH Dependent Cocrystal Stability and Solubility. Cryst. Growth Des. 2009, 9 (1), 378-385.

(7) Goud, N. R.; Suresh, K.; Sanphui, P.; Nangia, A. Fast Dissolving Eutectic Compositions of Curcumin. Int. J. Pharm. 2012, 439 (1-2), $63-72$.

(8) Cherukuvada, S.; Nangia, A. Eutectics as Improved Pharmaceutical Materials: Design, Properties and Characterization. Chem. Commun. 2014, 50 (8), 906-923.

(9) Hart, M. L.; Do, D. P.; Ansari, R. A.; Rizvi, S. A. A. Brief Overview of Various Approaches to Enhance Drug Solubility. J. Dev. Drugs 2013, $2(3), 1-7$.

(10) Gala, U.; Pham, H.; Chauhan, H. Pharmaceutical Applications of Eutectic Mixtures. J. Dev. Drugs 2013, 2 (3), 1-2.

(11) Blagden, N.; de Matas, M.; Gavan, P. T.; York, P. Crystal Engineering of Active Pharmaceutical Ingredients to Improve Solubility and Dissolution Rates. Adv. Drug Delivery Rev. 2007, 59 (7), 617-630.

(12) Domingos, S.; Andre, V.; Quaresma, S.; Martins, I. C. B.; Minas da Piedade, M. F.; Duarte, M. T. New Forms of Old Drugs: Improving without Changing. J. Pharm. Pharmacol. 2015, 67 (6), 830-846.

(13) Alhalaweh, A.; Sokolowski, A.; Rodríguez-Hornedo, N.; Velaga, S. P. Solubility Behavior and Solution Chemistry of Indomethacin Cocrystals in Organic Solvents. Cryst. Growth Des. 2011, 11 (9), 39233929.

(14) Kuminek, G.; Cao, F.; Bahia de Oliveira da Rocha, A.; Gonçalves Cardoso, S.; Rodríguez-Hornedo, N. Cocrystals to Facilitate Delivery of Poorly Soluble Compounds Beyond-Rule-of-5. Adv. Drug Delivery Rev. 2016, 101, 143.

(15) Perlovich, G. L.; Manin, A. N. Design of Pharmaceutical Cocrystals for Drug Solubility Improvement. Russ. J. Gen. Chem. 2014, 84 (2), 407-414.

(16) Kuminek, G.; Rodríguez-Hornedo, N.; Siedler, S.; Rocha, H. V. A.; Cuffini, S. L.; Cardoso, S. G. How Cocrystals of Weakly Basic Drugs and Acidic Coformers Might Modulate Solubility and Stability. Chem. Commun. 2016, 52 (34), 5832-5835.

(17) Caira, M. R.; Bourne, S. A.; Samsodien, H.; Engel, E.; Liebenberg, W.; Stieger, N.; Aucamp, M. Co-Crystals of the Antiretroviral Nevirapine: Crystal Structures, Thermal Analysis and Dissolution Behaviour. CrystEngComm 2012, 14 (7), 2541-2551.

(18) Goldberg, A. H.; Gibaldi, M.; Kanig, J. L. Increasing Dissolution Rates and Gastrointestinal Absorption of Drugs Vid Solid Solutions and Eutectic Mixtures III - Experimental Evaluation of GriseofulvinSuccinic Acid Solid Solution. J. Pharm. Sci. 1966, 55 (5), 487-492.

(19) Thipparaboina, R.; Thumuri, D.; Chavan, R.; Naidu, V. G. M.; Shastri, N. R. Fast Dissolving Drug-Drug Eutectics with Improved Compressibility and Synergistic Effects. Eur. J. Pharm. Sci. 2017, 104, $82-89$.

(20) Avula, S. G.; Alexander, K.; Riga, A. Predicting Eutectic Behavior of Drugs and Excipients by Unique Calculations. J. Therm. Anal. Calorim. 2010, 99 (2), 655-658.

(21) Rodríguez-Hornedo, N. Cocrystals: Molecular Design of Pharmaceutical Materials. Mol. Pharmaceutics 2007, 4 (3), 299-300.

(22) Kumar, S.; Nanda, A. Pharmaceutical Cocrystals: An Overview. Indian J. Pharm. Sci. 2017, 79 (6), 858-871.

(23) Stott, P. W.; Williams, A. C.; Barry, B. W. Transdermal Delivery from Eutectic Systems: Enhanced Permeation of a Model Drug, Ibuprofen. J. Controlled Release 1998, 50 (1-3), 297-308.

(24) Fucke, K.; Myz, S. A.; Shakhtshneider, T. P.; Boldyreva, E. V.; Griesser, U. J. How Good Are the Crystallisation Methods for CoCrystals? A Comparative Study of Piroxicam. New J. Chem. 2012, 36 (10), 1969-1977.

(25) Nalte, Y. K.; Arsul, V. A.; Shep, S. G.; Bothara, S. B. Solubility Enhancement of Nevirapine by Cocrystallisation Technique. J. Pharm. Res. 2015, 9 (8), 556-561.

(26) Fábián, L. Cambridge Structural Database Analysis of Molecular Complementarity in Cocrystals. Cryst. Growth Des. 2009, 9 (3), 14361443.

(27) Galek, P. T. A.; Fábián, L.; Motherwell, W. D. S.; Allen, F. H.; Feeder, N. Knowledge-Based Model of Hydrogen-Bonding Propensity 
in Organic Crystals. Acta Crystallogr., Sect. B: Struct. Sci. 2007, B63 (5), $768-782$.

(28) Cullity, B. D.; Stock, S. R. Elements of X-Ray Diffraction, 3rd ed.; Prentice Hall, 2001.

(29) Dinnebier, R. E.; Billinge, S. J. L. Powder Diffraction: Theory and Practice, 1st ed.; RCS Publishing, 2008.

(30) Carvalho, A. M. G.; Araújo, D. H. C.; Canova, H. F.; Rodella, C. B.; Barrett, D. H.; Cuffini, S. L.; Costa, R. N.; Nunes, R. S. X-Ray Powder Diffraction at the XRD1 Beamline at LNLS. J. Synchrotron Radiat. 2016, 23 (6), 1501.

(31) APEX3 Software; Bruker AXS Inc.: Madison, Wisconsin, USA, 2016.

(32) Sheldrick, G. M. Crystal Structure Refinement with SHELXL. Acta Crystallogr., Sect. C: Struct. Chem. 2015, 71 (1), 3-8.

(33) Metz, G.; Wu, X. L.; Smith, S. O. Ramped-Amplitude Cross Polarization in Magic-Angle-Spinning NMR. J. Magn. Reson., Ser. A 1994, 110, 219-227.

(34) Harris, R. K. Nuclear Magnetic Resonance Spectroscopy: A Physicochemical View; Longman Scientific \& Technical: Essex, UK, 1986.

(35) Fung, B. M.; Khitrin, A. K.; Ermolaev, K. An Improved Broadband Decoupling Sequence for Liquid Crystals and Solids. J. Magn. Reson. 2000, 142, 97-101.

(36) Harris, R. K. Nuclear Magnetic Resonance Spectroscopy; Longman Publishing Group: London, 1986.

(37) Murugan, N. A.; Sayeed, A. Thermal Behavior of Disordered Phase of Caffeine Molecular Crystal: Insights from Monte Carlo Simulation Studies. J. Chem. Phys. 2009, 130 (20), 204514.

(38) Caira, M. R.; Stieger, N.; Liebenberg, W.; De Villiers, M. M.; Samsodien, H. Solvent Inclusion by the Anti-HIV Drug Nevirapine: XRay Structures and Thermal Decomposition of Representative Solvates. Cryst. Growth Des. 2008, 8 (1), 17-23.

(39) Zakeri-Milani, P.; Barzegar-Jalali, M.; Azimi, M.; Valizadeh, H. Biopharmaceutical Classification of Drugs Using Intrinsic Dissolution Rate (IDR) and Rat Intestinal Permeability. Eur. J. Pharm. Biopharm. 2009, 73 (1), 102-106.

(40) Dezani, A. B.; Dezani, T. M.; Ferreira, J. C. F.; Serra, C. H. dos R. Solubility Evaluation of Didanosine: A Comparison between the Equilibrium Method and Intrinsic Dissolution for Biopharmaceutics Classification Purposes. Braz. J. Pharm. Sci. 2017, 53 (2), e16128.

(41) Löbmann, K.; Flouda, K.; Qiu, D.; Tsolakou, T.; Wang, W.; Rades, T. The Influence of Pressure on the Intrinsic Dissolution Rate of Amorphous Indomethacin. Pharmaceutics 2014, 6 (3), 481-493.

(42) Fandaruff, C.; Rauber, G. S.; Araya-Sibaja, A. M.; Pereira, R. N.; de Campos, C. E. M.; Rocha, H. V. A.; Monti, G. A.; Malaspina, T.; Silva, M. A. S.; Cuffini, S. L. Polymorphism of Anti-HIV Drug Efavirenz: Investigations on Thermodynamic and Dissolution Properties. Cryst. Growth Des. 2014, 14, 4968-4975. 